\title{
Phases of correlated spinless fermions on the honeycomb lattice
}

\author{
Maria Daghofer ${ }^{1}$ and Martin Hohenadler ${ }^{2}$ \\ ${ }^{1}$ Institut für Theoretische Festkörperphysik, IFW Dresden, 01171 Dresden, Germany \\ ${ }^{2}$ Institut für Theoretische Physik und Astrophysik, Universität Würzburg, 97074 Würzburg, Germany
}

(Dated: August 24, 2018)

\begin{abstract}
We use exact diagonalization and cluster perturbation theory to address the role of strong interactions and quantum fluctuations for spinless fermions on the honeycomb lattice. We find quantum fluctuations to be very pronounced both at weak and strong interactions. A weak second-neighbor Coulomb repulsion $V_{2}$ induces a tendency toward an interaction-generated quantum anomalous Hall phase, as borne out in mean-field theory. However, quantum fluctuations prevent the formation of a stable quantum Hall phase before the onset of the charge-modulated phase predicted at large $V_{2}$ by mean-field theory. Consequently, the system undergoes a direct transition from the semimetal to the charge-modulated phase. For the latter, charge fluctuations also play a key role. While the phase, which is related to pinball liquids, is stabilized by the repulsion $V_{2}$, the energy of its low-lying charge excitations scales with the electronic hopping $t$, as in a band insulator.
\end{abstract}

PACS numbers: 71.10.Fd,71.27.+a,71.30.+h,75.25.Dk

\section{INTRODUCTION}

Interest in topological states of matter has been boosted by the discovery of topological insulators and superconductors $\frac{112}{12}$ The quantum spin Hall insulator, a novel topological state of matter with a $Z_{2}$ topological invariant and helical edge states ${ }^{3 / 4}$ has been observed experimentally in $\mathrm{HgTe}$ quantum well structures $\stackrel{516}{{ }^{5}}$ Since then, research has broadened substantially, and now includes three-dimensional topological insulators and superconductors, ${ }^{2}$ fractional Chern insulators, ${ }^{78}$ and symmetry-protected topological phases $\frac{9}{14}$

Topological insulators and Chern insulators typically arise from complex hopping terms related to spin-orbit coupling ${ }^{3 / 4}$ or to a periodic vector potential ${ }^{[15}$ By now, several noninteracting models are known that support quantum Hall and quantum spin Hall phases $\frac{16}{16}$ In such settings, electron-electron interactions play a minor role as a result of the bulk band gap, and the states closely resemble band insulators. Sufficiently strong interactions can drive transitions to nontopological phases with magnetic ${ }^{17}\left[19\right.$ or charge-density-wave order. ${ }^{20}[22$ An interesting interaction-driven transition from a quantum spin Hall (QSH) phase to a phase with fractional excitations and topological order is the so-called $\mathrm{QSH}^{*}$ phase found in a mean-field treatment of a model for $\mathrm{Na}_{2} \mathrm{IrO}_{3}{ }^{23}$ The interplay of topological band structures and electronic interactions has been studied extensively, see Ref. 16 for a review.

Conversely, electronic correlations can also give rise to topological states. Topological Mott insulators, ${ }^{24}$ or interaction-generated topological insulators, are a particularly interesting concept. Raghu et al. ${ }^{24}$ presented a scenario where quantum (spin) Hall states arise purely from electronic interactions that give rise to spontaneously generated, complex bond order parameters. ${ }^{24 \mid 25}$ Such a correlation-driven route to topological states would abandon the requirement of strong intrinsic spin-orbit coupling and could thus significantly extend the class of topologically nontrivial materials. In a more general context, correlations have been shown to stabilize topologically nontrivial bands in double-exchange models on the kagome and triangular lattices, $\frac{26127}{2}$ where the topological character is supported by the coupling to localized spins. In particular, it has been shown that Haldane's scenario of a transition from bands featuring Dirac cones to bands with a topologically nontrivial gap can be observed in the Kondo-lattice model on the checkerboard lattice $\sqrt{28}$ The ordering of complex orbitals can also lead to topological insulators. 29,32

For the spinless model considered in Ref. 24, the existence of a topological phase, namely a quantum anomalous Hall (QAH) state, has been confirmed by more elaborate mean-field approximations. ${ }^{33 / 34}$ More generally, again using mean-field and renormalization group methods, interaction-generated topological states have been shown to arise in kagome, checkerboard, or decorated honeycomb lattices, 35 in a $\pi$-flux square lattice model,$\stackrel{33}{, 3}$ as well as in three dimensions. ${ }^{[38}$ In contrast to checkerboard and kagome lattices, the Dirac points in the $\pi$-flux and honeycomb model are associated with a vanishing density of states at the Fermi level. Consequently, transitions to symmetry-broken phases may not be correctly captured by a weak-coupling approach. The variety and fascinating properties of these novel phases make it desirable to go beyond a weak coupling description. Indeed, recent exact diagonalization results ${ }^{39}$ for the $\pi$-flux square lattice model have not confirmed mean-field predictions of an interaction-generated QAH phase.$^{33}$

Here, we use exact diagonalization to study the spinless model first considered in Ref. 24. Thereby, we fully take into account quantum fluctuations which are expected to be strong given the low coordination number of the honeycomb lattice. Most importantly, our results imply that the interaction-generated topological mean-field state is unstable with respect to fluctuations, and that the gapped ground state is not adiabatically connected to the QAH state of the Haldane model. However, we 
demonstrate that for small $V_{2}$, the model has a tendency toward an interaction-generated QAH state. In addition, we provide new insights into the charge-ordered phase that exists for strong next-nearest-neighbor repulsion. 34 The organization of this paper is as follows. In Sec. III we define the model considered. Our results are discussed in Sec. III, and we conclude in Sec. IV]

\section{MODEL}

Following Ref. 24, we consider a model of interacting, spinless fermions described by the Hamiltonian

$$
\hat{H}_{1}=\hat{H}_{0}+V_{1} \sum_{\langle i j\rangle} \hat{n}_{i} \hat{n}_{j}+V_{2} \sum_{\langle\langle i j\rangle\rangle} \hat{n}_{i} \hat{n}_{j} .
$$

The first term, $\hat{H}_{0}=-t \sum_{\langle i j\rangle}\left(c_{i}^{\dagger} c_{j}+c_{j}^{\dagger} c_{i}\right)$, describes nearest-neighbor (NN) hopping on the honeycomb lattice. The second term accounts for a repulsion between fermions on NN sites, whereas the third term describes a repulsion between next-nearest-neighbor $(\mathrm{NNN})$ sites (i.e., sites on the same sublattice). The indices $i, j$ number lattice sites, and $L$ denotes the total number of sites. Throughout this paper, we consider a half-filled band with one fermion per unit cell and $\left\langle n_{i}\right\rangle=1 / 2$.

Hamiltonian (1) was previously studied at the meanfield level.2433134 These works reported a QAH state with chiral edge states and a nonzero Chern index. This phase is characterized by a complex bond order parameter ${ }^{24}$ $\chi_{i j}=\chi_{j i}^{*}=\left\langle c_{i}^{\dagger} c_{j}\right\rangle$ that mimics the complex hopping term of the Haldane mode ${ }^{15}$ and breaks time-reversal symmetry. The QAH state is driven by $V_{2}$ and, according to mean-field theory, most stable for $V_{1}=0.2433[34$ For $V_{1}=0$, Refs. 24 and 33 found a semimetal (SM) and a QAH phase. The SM is stable up to a finite critical value of $V_{2}$ because of the vanishing density of states at the Fermi level. Using a more elaborate mean-field ansatz, Grushin et al ${ }^{\text {[34 }}$ obtained an additional, chargemodulated $(\mathrm{CM})$ insulating phase at large $V_{2} / t$ that restricts the QAH phase to a finite region $1.5 \lesssim V_{2} / t \lesssim 2.5$. For $V_{1}>0$, a charge-density-wave phase with broken inversion symmetry 24133134 (for $V_{1}>V_{2}$ ), as well as a Kekulé ordered phase with broken translational invariance (for $V_{1} \sim V_{2}$ ) were found. $\stackrel{33134 \mid 40}{ }$ The low-energy field theory of interacting spinless fermions on the honeycomb lattice is discussed in Ref. 41.

\section{RESULTS}

According to mean-field theory, $24 \mid 33134$ the QAH phase is stabilized by $V_{2}$, and is therefore most extended in parameter space for $V_{1}=0$. Therefore, and to simplify the analysis, we focus on the case $V_{1}=0$, although some results for nonzero $V_{1}$ will also be presented.

The exact diagonalization results presented below have been obtained on clusters with 18,24 , and 30 sites, re- spectively. Since the Dirac points $\pm K$ define the lowenergy physics of the noninteracting system $\left(V_{1}=V_{2}=\right.$ 0 ), and also correspond to the ordering wavevector of the charge order driven by large values of $V_{2}$ (see below), we have chosen clusters for which $\boldsymbol{q}= \pm K$ are allowed momenta. In the notation of Ref. 20, the clusters used here correspond to $18 \mathrm{~A}$ and as well as $24 \mathrm{~A}$; results for a small number of parameter sets were also obtained using 30A. We have verified that our findings are unchanged when using clusters $24 \mathrm{C}$ and $24 \mathrm{D}$.

\section{A. Phase diagram for $V_{1}=0$}

The QAH state found in mean-field theory $24[33[34$ is identical to the $\mathrm{QAH}$ ground state of the noninteracting Haldane model,$\frac{15}{, 5}$ and hence characterized by a Chern number $C= \pm 1$. To prove the existence of this phase numerically, it is not sufficient to simply calculate the Chern index for the model (1). The reason is that there exist two possible bond-order patterns which differ by an overall sign, and describe Chern insulators with $C=1$ and $C=-1$, respectively. When the ground state of a finite cluster is determined by exact diagonalization, it can be expected to be a linear combination of these two states, and hence to have a vanishing Chern index. Finally, the accessible system sizes are not sufficient to carry out a finite-size extrapolation to the thermodynamic limit to reveal a symmetry breaking. Given these complications, a different route has to be chosen.

To identify a possible QAH state driven by the interaction $V_{2}$, we here study a superposition $\underline{42}$ of the Hamiltonian of interest, namely Eq. (1), and a Hamiltonian known to have the QAH ground state predicted by meanfield theory. The mean-field QAH state of Eq. (1), first reported in Ref. 24, is identical to the QAH state of the Haldane Hamiltoniani 15

$$
\hat{H}_{2}=\hat{H}_{0}-t_{2} \sum_{\langle\langle i j\rangle\rangle}\left(e^{\mathrm{i} \phi_{i j}} c_{i}^{\dagger} c_{j}+e^{-\mathrm{i} \phi_{i j}} c_{j}^{\dagger} c_{i}\right),
$$

for the choice of phase $\phi_{i j}= \pm \pi / 2$; the sign depends on the direction of the bond $\langle\langle i j\rangle\rangle$ and the sublattice. It arises from periodic magnetic fluxes that sum to zero for each hexagon of the honeycomb lattice $\frac{15}{}$ The hopping term $\hat{H}_{0}$ is identical to Eq. (1). Hence, at the mean-field level, the Hamiltonian

$$
\widetilde{H}(\lambda)=\lambda H_{2}+(1-\lambda) H_{1}, \quad \lambda \in[0,1],
$$

interpolates between the noninteracting QAH ground state of the Haldane Hamiltonian $\hat{H}_{2}$ (i.e., $t_{2}>0, V_{2}=0$, $\lambda=1$ ) and the interaction-generated $\mathrm{QAH}$ ground state of $\hat{H}_{1}\left(\lambda=0\right.$, and suitable values of $\left.V_{2}\right)$. As a function of $\lambda$, it is therefore possible to adiabatically connect the ground states that exist for $\lambda=0$ and $\lambda=1$. For $0<\lambda<1, \widetilde{H}(\lambda)$ describes interacting fermions on the honeycomb lattice with additional Haldane hopping $t_{2}$. 
If the mean-field QAH state is stable, a similar adiabatic connection between $\lambda=0$ and $\lambda=1$ is expected to exist when $\widetilde{H}(\lambda)$ is solved using exact numerical methods. Starting with $\lambda=0$, we hence expect a continuous evolution with $\lambda$ if the ground state of $\hat{H}_{1}$ is indeed a QAH state. In particular, switching on $t_{2}$ should reinforce a potential QAH ground state of $\hat{H}_{1}$. Conversely, a discontinuous evolution (e.g., a phase transition) as a function of $\lambda$ would imply that the state at $\lambda=0$ is not the QAH state predicted by mean-field theory.

Here, we calculate the quantum fidelity $F=$ $\left\langle\phi_{0}\left(V_{2}+\delta V_{2}\right) \mid \phi_{0}\left(V_{2}\right)\right\rangle$, corresponding to the overlap of the ground states of Hamiltonian (1) for $V_{2}$ and $V_{2}+\delta V_{2}$, respectively, with all other parameters unchanged. The fidelity permits us to detect transitions between different phases without making assumptions regarding order parameters ${ }^{43144}$ Moreover, it is particularly suitable to detect transitions between topologically trivial and nontrivial insulators, 45 because such transitions involve a level crossing even on finite clusters. ${ }^{20 \mid 21]}$ In contrast, continuous symmetry-breaking transitions appear as gradual changes on finite clusters, and are therefore often difficult to identify.

We take the mean-field phase diagram as a starting point, and distinguish three regimes. For sufficiently large $V_{2}$ (the mean-field prediction is $V_{2} \gtrsim 2.5 t$ ), the gapped $\mathrm{CM}$ phase is expected ${ }^{34}$ For smaller $V_{2}(1.5 \lesssim$ $V_{2} / t \lesssim 2.5$, according to Ref. 34), the QAH state exists, and for $V_{2} \lesssim 1.5 t$, mean-field theory finds the SM phase.

We first consider the $\mathrm{CM}$ region. In Fig. 1(a), we show the evolution of the two lowest energy levels of $\widetilde{H}(\lambda)$ along a path from $\left(t_{2}, V_{2}\right)=(0.3 t, 0)(\lambda=1)$ to $\left(t_{2}, V_{2}\right)=(0,4 t)(\lambda=0)$, as indicated by the solid line in Fig. 1 (b). Whereas the point $\lambda=1$ lies in the wellestablished QAH phase of the Haldane model, the point $\lambda=0$ has a sufficiently large $V_{2}$ to fall into the CM phase .34 [The existence of charge order will be demonstrated below, see Fig. 2(a).] Since $\lambda=1$ corresponds to the Haldane model, the initially lower-lying level (solid line) in Fig. 1(a) can be identified with the QAH state with Chern number $C=1$. We find that switching on $V_{2}$ in the Haldane model does not immediately destroy the QAH state, as can be expected for a gapped phase. However, at a critical value $V_{2} \approx 2.9 t$ (and $t_{2} \approx 0.08 t$ ) we observe a level crossing within the same momentum sector, and a vanishing of the fidelity. This level crossing, signaling a quantum phase transition to a topologically distinct state, reveals that the QAH state at $t_{2}=0.3 t, V_{2}=0$ is different from the gapped ground state at $t_{2}=0, V_{2}=4 t$, in accordance with the mean-field theory prediction of a CM phase for these parameters.

Figure 1(b) shows the level crossings found along similar paths in the $\left(t_{2}, V_{2}\right)$ plane, but with end points that have different values of $V_{2}$. For values as small as $V_{2}=2.5 t$, we find the same type of level crossing as illustrated in Fig. 1(a). Moreover, with decreasing $V_{2} / t$, the level crossings move toward smaller values of $t_{2}$, in accordance with the decrease of the gap of the CM state.
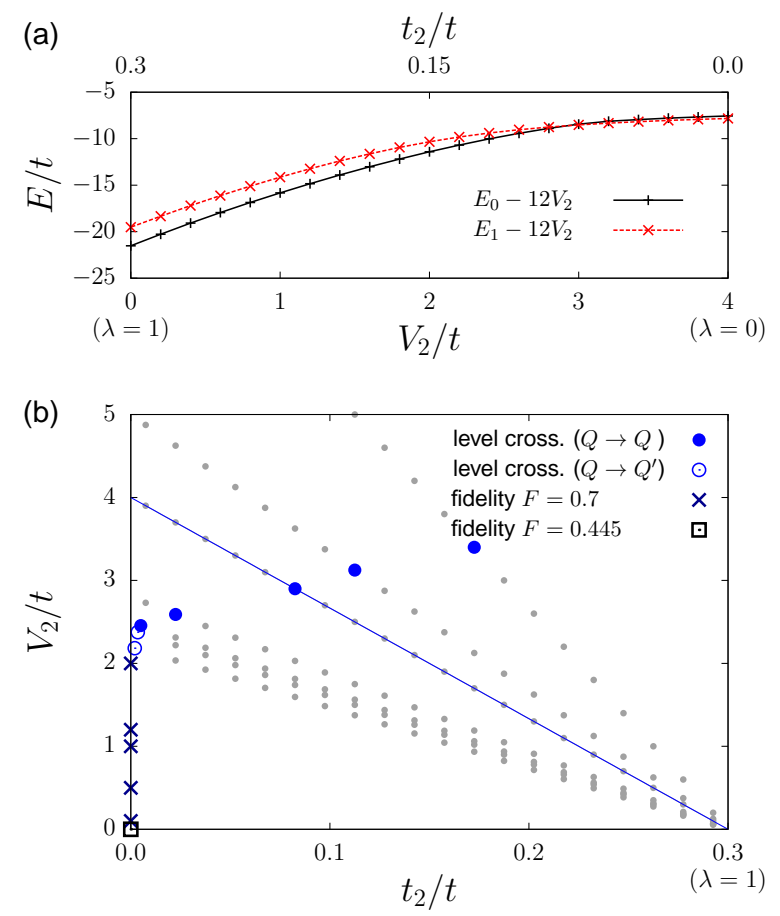

(c)

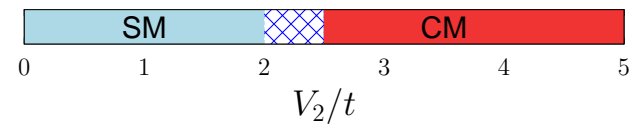

FIG. 1. (Color online) (a) The two lowest energy levels in the ground state momentum sector along the path from $\left(t_{2}=0.3, V_{2}=0\right)$ to $\left(t_{2}=0, V_{2}=4\right)$, indicated by the solid line in (b). (b) Moving along the parameter trajectories indicated by the small dots from the QAH state of the Haldane model (2) at $t_{2}=0.3 t, V_{2}=0(\lambda=1)$ toward the model (1) with $t_{2}=0$ and different $V_{2}(\lambda=0)$, we find level crossings at the points indicated by large circles. Large open (filled) circles correspond to level crossings with (without) a change of the ground-state momentum sector. Results in (a) and (b) are for $V_{1}=0$. (c) Phase diagram of Hamiltonian (1) with $V_{1}=0$. The hatched region reflects the uncertainty regarding the critical point due to finite-size effects. All results were obtained from exact diagonalization of $\widetilde{H}$ using a 24-site cluster.

In the regime $2 t \lesssim V_{2} \lesssim 2.5 t$, a level crossing occurs at small but finite values of $t_{2}$, but between different ground-state momentum sectors (indicated by open circles). Moreover, the ground state in this regime is doubly degenerate with momenta $\pm K$, as opposed to the nondegenerate ground state with momentum $\Gamma$ that exists for $V_{2} / t$ outside $[2,2.5]{ }^{46}$ This behavior can be understood by considering the model with $t_{2}=0$, see Fig. 2(a) and discussion below, for which we observe two changes of the momentum sector as a function $V_{2}$ at $V_{2} \approx 2 t(\Gamma \rightarrow \pm K)$ and $V_{2} \approx 2.5 t( \pm K \rightarrow \Gamma)$. We attribute the existence of this intermediate regime and the momentum changes to finite-size effects related to the close energetic proximity of excited states with momentum $\boldsymbol{q}= \pm K$ to the ground state in the SM and the CM phases. Indeed, the 
momentum-changing level crossings are absent on $L=18$ and $L=30$ clusters, and a similar cluster-dependent intermediate region has been reported for the interacting Haldane model. ${ }^{21}$ More importantly, the fact that a level crossing occurs as a function of $\lambda$ implies that the ground state of $\hat{H}_{1}$ is not adiabatically connected to the QAH state of the Haldane model down to $V_{2} \approx 2 t$.

Finally, for interactions $V_{2} \lesssim 2 t$, the fidelity $F=$ $\left\langle\phi_{0}\left(V_{2}, t_{2}\right) \mid \phi_{0}\left(V_{2}, t_{2}+\delta t_{2}\right)\right\rangle$ with $\delta t_{2}>0$, which is very close to 1 for $t_{2}>0$, decreases to 0.7 (or $1 / \sqrt{2}$ ) for $t_{2}=0$; the corresponding parameters are indicated by the crosses in Fig. 1(b). The fact that any finite $t_{2}$ significantly modifies the ground state suggests that in this regime, we have the SM phase which is unstable toward the opening of a topological mass gap by a finite $t_{2}$. The same behavior can be observed in the noninteracting $\mathrm{Hal}$ dane model. ${ }^{[5]}$ We observe the same fidelity over the range $V_{2} \in[0,2 t]$, which suggests that the SM phase extends at least up to $V_{2}=2 t$. This value is comparable to the mean-field estimates. $24|33| 34$

The results of this section, in particular the fact that the gapped parameter region of Hamiltonian (1) cannot be adiabatically connected to the QAH state of the Haldane model (2), suggest that the mean-field prediction of a QAH phase is not borne out. Instead, we propose the $V_{1}=0$ phase diagram shown in Fig. 11(c), with a direct transition from the $\mathrm{SM}$ to the $\mathrm{CM}$ phase at a critical value $V_{2} \approx 2.5 t$. We will see below that this scenario is consistent with the results for charge structure factors [note the jump of $S(K)$ in Fig. 2 near $V_{2}=2.5 t$ ] and the density of states.

\section{B. Charge order driven by $V_{2}$}

Given a two-site unit cell, two charge structure factors $S^{ \pm}(\mathbf{q})$ can be defined for each sublattice momentum $\mathbf{q}$, which differ by the relative phase between the contributions of the two sublattices and can be written as

$\left.S^{ \pm}(\mathbf{q})=\frac{1}{L}\left|\sum_{j} \mathrm{e}^{i \mathbf{q} \cdot \mathbf{r}_{j}}\left[\left(\hat{n}_{j}^{A}-\frac{1}{2}\right) \pm\left(\hat{n}_{j}^{B}-\frac{1}{2}\right)\right]\right| \phi_{0}\right\rangle\left.\right|^{2}$.

Here, $\hat{n}_{j}^{\alpha}$ is the density operator for a site on sublattice $\alpha$ in unit cell $j$, and $\left|\phi_{0}\right\rangle$ denotes the many-body ground state. A Néel-type charge order corresponding to a sublattice charge imbalance within the unit cell, as previously observed for spinless fermions with interaction $V_{1}, 24$ is captured by $S^{-}(\Gamma)$ with $\Gamma=(0,0)$, whereas the charge order predicted by mean-field theory ${ }^{34}$ for large $V_{2}$ can be tracked by $S(K) \equiv \frac{1}{2}\left[S^{+}(K)+S^{-}(K)\right]$.

Figure 2 shows results for these structure factors for 24 and 18-site clusters, obtained for the original model (1). The Néel structure factor $S^{-}(\Gamma)$ is quickly suppressed from its noninteracting value with increasing $V_{2}$, while $S(K)$ is enhanced. The open circles in Fig. 2 indicate where the ground state of the $L=24$ cluster changes momentum, see discussion above. Whereas $S(K)$ continues to grow in this regime, $S^{-}(\Gamma)$ is almost unchanged.
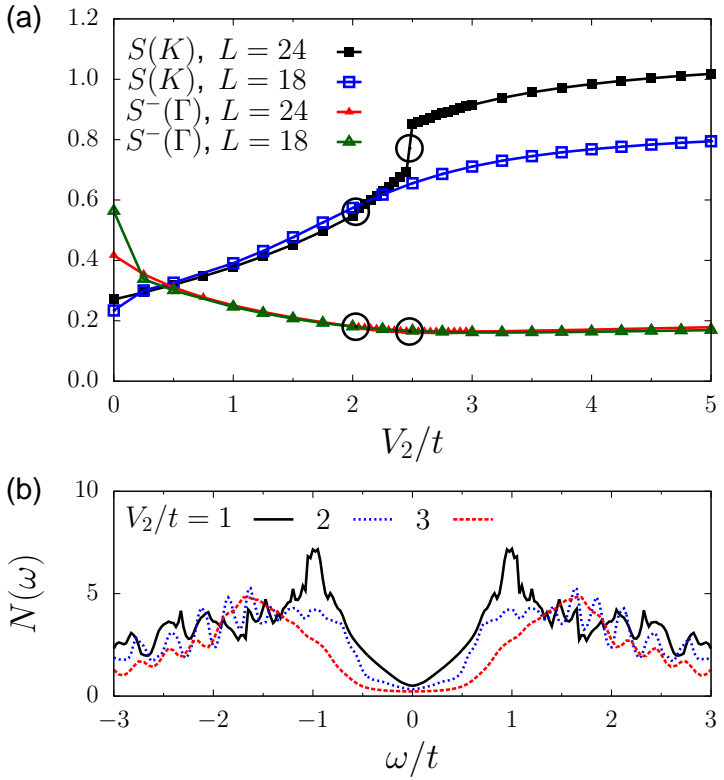

FIG. 2. (Color online) (a) Charge structure factors $S(K)$ and $S^{-}(\Gamma)$, see Eq. (4), from exact diagonalization for two different cluster sizes $(L=18$ and $L=24)$. The open circles at $V_{2} \approx 2 t$ and $V_{2} \approx 2.5 t$ indicate the change of the ground state momentum sector observed for $L=24$. (b) Density of states for different values of $V_{2} / t$ obtained from exact diagonalization with twisted boundary conditions and $L=24$. All results are for $V_{1}=0$.

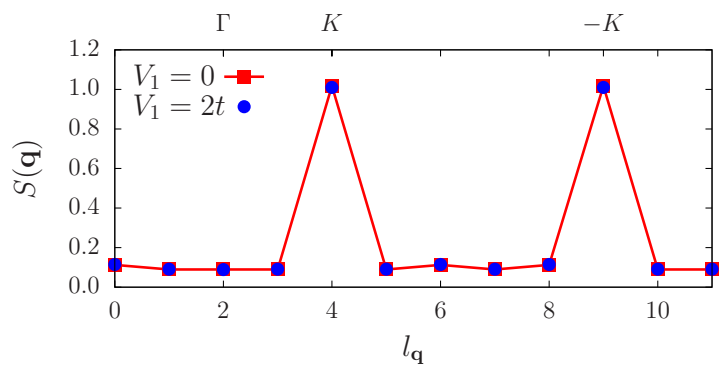

FIG. 3. (Color online) Charge structure factor $S(\boldsymbol{q})$ for $L=24$ sites, $t_{2}=0, V_{2}=5 t$ (deep in the CM phase), and two values of the NN Coulomb repulsion $V_{1}$. Here, $l_{\mathbf{q}}$ indexes the wavevectors $\boldsymbol{q}$, with $l_{\boldsymbol{q}}=4,9$ corresponding to $\boldsymbol{q}= \pm K$ and $l_{\boldsymbol{q}}=2$ corresponding to $\boldsymbol{q}=\Gamma$. Since $S^{+}(\Gamma)=0, S(\Gamma)$ is proportional to the Néel structure factor $S^{-}(\Gamma)$.

This finding suggests that this intermediate regime is not a different phase, since in that case we would expect the charge order [i.e., $S(K)$ ] to be suppressed. Other potential order parameters that we considered (including bond order) are similarly unaffected in this parameter region. Moreover, while a finite $t_{2}$ is needed to move from this regime to the QAH state, the very small critical values (e.g., $t_{2}=0.003 t$ for $V_{2}=2.3 t$ ) are a strong argument against any gapped intermediate phase.

These results, together with the absence of an intermediate region where the ground state momentum changes 
on the $L=18$ and $L=30$ clusters, agree with our previous conclusion, namely a direct transition from the SM to the CM phase. Keeping in mind that a finite-size scaling is not feasible with the accessible cluster sizes, our estimate for the critical point is $V_{2} \approx 2.5 t$. Figure $2(\mathrm{~b})$ shows the density of states obtained for a 24 -site cluster. Our use of twisted boundary conditions reduces finitesize effects by enhancing the resolution in momentum space, but can induce small spurious gaps as a result of the breaking of translational symmetry ${ }^{47}$ Nevertheless, the results in Fig. 2(b) suggest the existence of a singleparticle gap for $V_{2} \gtrsim 2.5 t$, in accordance with the phase diagram shown in Fig. 1 (c).

An insulating, charge-ordered phase at large values of $V_{2} / t$ (referred to as the CM phase) was first observed in Ref. 34, and a related charge-ordered phase has been reported for spinful fermions in honeycomb bilayers and trilayers $\frac{48149}{}$ At the mean-field level, the pattern of charge density deviations from half filling takes the form $+\delta,-\delta,+\delta,+\Delta,-\Delta,-\delta$ (with $\Delta>\delta$ ) for consecutive sites of the hexagonal unit cell ${ }^{34}$ Within each sublattice, charge is modulated with a three-site unit cell, corresponding to an ordering wavevector $K$, and charge is in general unequally distributed between the sublattices (except for $\Delta=2 \delta$ ). The CM phase is different from the charge-density-wave phase with a Néel-type charge modulation which is driven by large values of $V_{1}$.24

Our exact diagonalization results shown in Fig. 2 suggest a transition from the SM to the $\mathrm{CM}$ phase at $V_{2} \gtrsim 2.5 t$. The fact that the Néel structure factor $S^{-}(\boldsymbol{q})$ remains comparable to $S(\boldsymbol{q})$ at other momenta $\boldsymbol{q} \neq \pm K$, see Fig. 3. provides an argument against charge imbalance between the sublattices. On the other hand, the Néel signal $S^{-}(\Gamma)$ is not suppressed when we move deeper into the CM phase (larger $V_{2} \gg 2 t$ ) either, see Fig. 2(a), as one would expect for the mean-field phase with $\Delta=2 \delta$.

A closer analysis reveals that the CM phase driven by $V_{2}$ has an intrinsically fluctuating and partly disordered character, which is due to geometric frustration and cannot be fully captured by simple mean-field approaches. $\underline{50}$ The origin of these fluctuations becomes apparent by considering the atomic limit $t=0$, in which the honeycomb lattice decouples into two triangular lattices, on each of which fermions experience the frustrated repulsion $V_{2}$. Figure 4(a) illustrates the charge distribution on one sublattice. Filled (empty) circles correspond to occupied (empty) sites, contributing 1/6 (1/3 when we consider both sublattices) to the total band filling of $1 / 2$. The remaining fermions can be distributed over the $\oplus$ sites at an energy cost of $3 V_{2}$ each. Since all possible configurations have the same classical energy, a highly degenerate ground state with only partial charge order results. An analogous situation was analyzed for the Ising model on the triangular lattice by Wannier ${ }^{[51]}$ Interestingly, the energy is also independent of the relative number of occupied $\oplus$ sites on the two sublattices. Consequently, the repulsion $V_{2}$ is equally satisfied by configurations with (a)

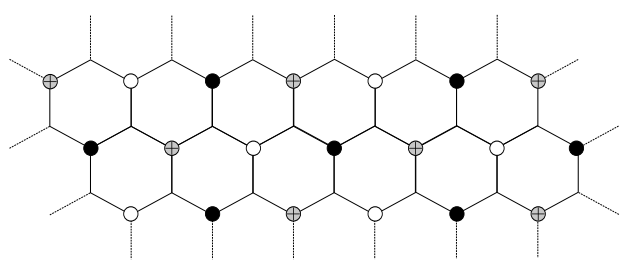

(b)

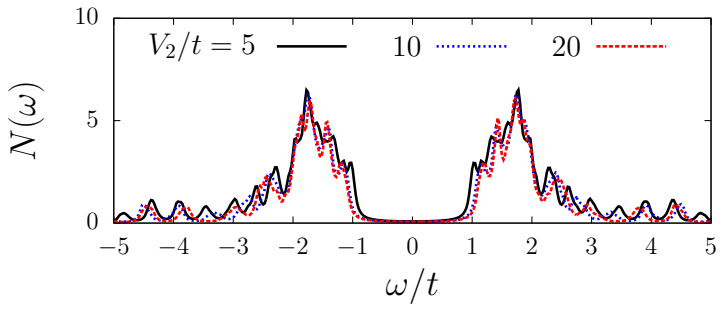

FIG. 4. (Color online) (a) Atomic-limit charge distribution for one of the two sublattices. Filled (empty) circles indicate occupied (empty) lattice sites. Any distribution of the remaining fermions over the $\oplus$ sites gives the same energy. (b) Density of states in the CM phase from exact diagonalization with twisted boundary conditions and $L=24$.

fermions evenly distributed among the two sublattices, and configurations with ratios $1: 2$ or $2: 1$, or anything in between. In fact, even a nonzero $\mathrm{NN}$ repulsion $V_{1}$ leaves the degeneracy largely intact (the energy cost for fermions on $\oplus$ sites becomes $\left.3 V_{2}+V_{1}\right)$ and does in particular not favor charge imbalance between the sublattices, as shown in Fig. 3. The degeneracy can hence only be lifted by the hopping $t$.

For spinless fermions on the triangular lattice, it has been shown ${ }^{52}$ that the partial charge order persists also for a nonzero hopping $t \neq 0$. The extensive degeneracy due to disorder is there reduced to the three-fold degeneracy of the charge-order pattern, because the additional fermions form a metal. This metallic yet partially charge-ordered phase was dubbed a pinball liquid ${ }^{52}$ Depending on the Hamiltonian, the electrons not involved in charge order can show superconductivity ${ }^{53}$ or topological order ${ }^{[54}$ In the present model, the hopping $t$ connects the two sublattices, and can provide the largest kinetic energy gain when the densities in the two sublattices are equal. It will consequently tend to lift the degeneracy between different sublattice occupations in favor of equal occupancy. Indeed, the charge structure factor $S(\mathbf{q})$ shown in Fig. 3 does not indicate a charge imbalance between the sublattices: While $S(\mathbf{q})$ is clearly peaked at the ordering momenta $\pm K$, the Néel signal [corresponding to $l_{\mathbf{q}}=2$, see caption of Fig. 3 is not enhanced. Its weight and that for other momenta $\mathbf{q} \neq \pm K$ approach a nonzero value for large $V_{2}$. In contrast, the structure factor is suppressed to zero for momenta $\mathbf{q} \neq \Gamma$ deep in the charge-density-wave phase. These nonzero values of $S(\boldsymbol{q})$ for $\mathbf{q} \neq \pm K$ in the CM phase support the picture that not all fermions participate in the $K$-modulated charge order. Figure 3 also reveals that a finite (but moderate) $V_{1}=2 t$ does not increase the Néel signal, or indeed in- 
duce any significant changes, similar to the situation on decoupled sublattices.

In contrast to the metallic pinball liquid found in decoupled sublattices, $\frac{52}{\sqrt{22}}$ the density of states in Fig. 4(b) shows a gap for the CM phase at $V_{2}=3 t$. By comparing results for different values of $V_{2}$, we find that, after initially increasing with $V_{2}$, the gap saturates deep in the CM phase. [There are additional high-energy excitations on the scale of $V_{2}$ outside the energy range show in Fig. 4(b).] For large $V_{2}$, where the picture of two coupled pinball liquids is most applicable, the gap becomes independent of $V_{2}$ and instead scales with the hopping $t$. The analog of the metallic pinball liquid in the model (1) is therefore the insulating CM phase with interactionindependent low-energy excitations at $|\omega| \sim t$.

\section{Phases at nonzero $V_{1}$}

To establish the robustness of our findings at $V_{1}=$ 0 , we briefly consider a nonzero $V_{1}$. Figure 5 (a) shows results similar to Fig. 1(b), obtained for $\widetilde{H}$ by varying the parameters $t_{2}, V_{2}$ and $V_{1}$ along paths that connect the Hamiltonians $\hat{H}_{1}$ and $\hat{H}_{2}$. The starting point in the lower right corner corresponds to the QAH phase of the Haldane model $\hat{H}_{2}$ with $t_{2}=0.3$ and $V_{1}=V_{2}=0$. On the left vertical axis, $t_{2}=0, V_{1}=t$, and $V_{2}$ takes on the values indicated in the plot. (Starting from $\lambda=0$ and while switching off $t_{2}$, we switch on $V_{1}$ and $V_{2}$ at the same rate.) In addition, we have performed horizontal scans at fixed $V_{1}$ and $V_{2}$ starting from $t_{2}=0$.

For paths with $V_{2} \gtrsim 2.5 t$ at $\lambda=0$, we find level crossings in the same ground state momentum sector at finite critical values of $t_{2}$ (filled circles). As for Fig. 1(b), we interpret these crossings as quantum phase transitions and hence as the absence of an adiabatic connection between the ground state of $\hat{H}_{1}$ and the QAH phase of the Haldane model. Instead, the results for the charge structure factors in Fig. 5(b) suggest that the gapped phase at large $V_{2}$ is again the CM phase. As already seen in Fig. 3. the $V_{2}$-driven charge modulation is hardly affected by a small to moderate $V_{1}$. At intermediate values $2.1 \lesssim V_{2} / t \lesssim 2.5$, the same cluster-related complications arise as for Fig. 1(b), namely a level crossing with a change of the ground state momentum sector. As for $V_{1}=0$, see Fig. 1(b), level crossings take place at finite but very small $t_{2} \approx 0.003 t$. The $V_{2}$-driven charge modulations grow throughout this regime, see Fig. 5(b), and as argued in Sec. IIIB, we regard the level crossings as a finite-size effect rather than indications of an intermediate phase. The fact that $V_{1}$ has a negligible impact in this parameter regime can be taken as further evidence against potential intermediate phases that would be either stabilized or destabilized by a nonzero $V_{1}$.

For $1 \lesssim V_{2} / t \lesssim 2.1$, the fidelity jumps from $F \approx 1$ to $F \approx 0.7 \approx \sqrt{2}$ when $t_{2}$ becomes zero, indicating the existence of a gapless SM phase that is unstable towards the opening of a QAH gap via $t_{2}$. The only dif-
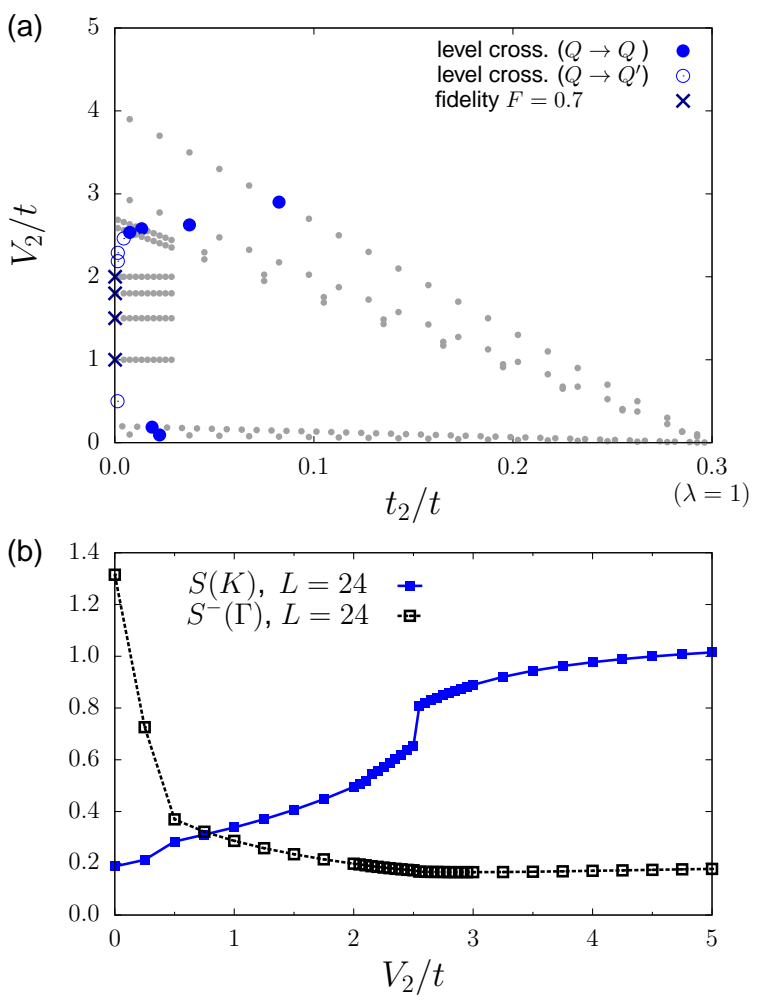

FIG. 5. (Color online) (a) As in Fig. 1(b), but for nonzero $V_{1}=t$. $V_{1}$ is switched on at the same rate as $V_{2}$, with $V_{1}=t$ along the left vertical axis. All results were obtained from exact diagonalization of $\widetilde{H}$ using a 24-site cluster. (b) Charge structure factors $S(K)$ and $S^{-}(\Gamma)$, see Eq. (4), from exact diagonalization with $L=24$.

ference to the case $V_{1}=0$ shown in Fig. 1 (b) is found at very small $V_{2}$, where we again see a level crossing with vanishing fidelity. In agreement with mean-field and numerical results, ${ }^{3455}$ these level crossings indicate the transition from the QAH phase at $V_{1}=0$ and $t_{2}>0$ to the gapped Néel charge-density-wave state found at $V_{1}=1, t_{2} \approx 0, V_{2} \approx 0$. The existence of the latter is also supported by the peak in $S^{-}(\Gamma)$ visible in Fig. 5(b). Between this regime with Néel order and the SM, we again observe a transition regime involving level crossings between different momentum sectors which we attribute to the cluster geometry.

Except for the vicinity of $V_{2}=0$, the results for $V_{1}=t$ are hence very similar to those for $V_{1}=0$. Consequently, our conclusion regarding the absence of an intermediate phase and a direct transition from the SM phase to the CM phase also holds at nonzero $V_{1}$ and is hence robust.

\section{Quantum fluctuation effects from cluster perturbation theory}

Our numerical results suggest a direct transition from the Dirac SM to the CM phase, and hence the absence of an intermediate QAH phase. A better understanding of 


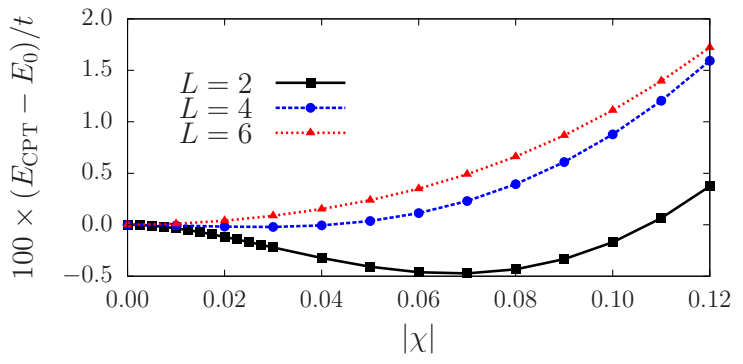

FIG. 6. (Color online) Ground-state energy as a function of the bond order parameter $\chi_{i j}= \pm \mathrm{i}|\chi|$ from cluster perturbation theory with different cluster sizes $L$. Here, $V_{2}=2 t$ and $V_{1}=0$. For $L=6$, the energy is minimal at $|\chi|=0$, corresponding to the absence of a QAH phase.

this issue can be obtained by systematically adding quantum fluctuations to the mean-field ansatz using a variant of cluster perturbation theory $[56]$ Within this approach, we treat interactions and hopping processes inside a finite cluster of $L$ sites exactly. Single-particle terms that connect different clusters, including the mean-field decoupled interaction terms, are accounted for in first-order perturbation theory $[57$

For the Hamiltonian (1), the mean-field decoupling reads $\hat{n}_{i} \hat{n}_{j} \mapsto\left\langle n_{i}\right\rangle \hat{n}_{j}+\hat{n}_{i}\left\langle n_{j}\right\rangle-\left\langle n_{i}\right\rangle\left\langle n_{j}\right\rangle-\left\langle c_{i}^{\dagger} c_{j}\right\rangle c_{j}^{\dagger} c_{i}-$ $\left\langle c_{j}^{\dagger} c_{i}\right\rangle c_{i}^{\dagger} c_{j}+\left|\left\langle c_{i}^{\dagger} c_{j}\right\rangle\right|^{2}$. The first three (Hartree) terms can give rise to charge-density-wave order, whereas the last three (Fock) terms can lead to bond-ordered phases. In particular, the QAH state emerges from an imaginary bond order parameter $\chi_{i j}=\left\langle c_{i}^{\dagger} c_{j}\right\rangle= \pm \mathrm{i}|\chi|$ with opposite sign on the two sublattices $\frac{58}{58}$ To explore the most favorable setting for the QAH phase, we set the chargedensity-wave order parameters $\left\langle n_{j}\right\rangle$ to zero and allow for purely imaginary $\chi_{i j}$ only. For a two-site cluster $(L=2)$ and $V_{1}=0$, cluster perturbation theory is equivalent to mean-field theory since all $V_{2}$ interaction terms are decoupled. Increasing $L$ allows for ordered patterns with a larger unit cell, similar to mean-field theory $\longdiv { 3 3 1 3 4 }$ and additionally includes short-range quantum fluctuations by treating more and more bonds exactly.

The impact of quantum fluctuations is apparent from Fig. 6 which shows the total energy as a function of $|\chi|$ for clusters with $L=2,4$, and 6 sites. Whereas the QAH state exists for $V_{2}=2 t$ in mean-field theory ${ }^{24|33| 34}$ and for $L=2$ in Fig. 6, it is quickly suppressed with increasing $L$. Already for $L=6$, the energy is minimal for $|\chi|=0$, and a QAH phase is absent.

\section{E. Tendency toward a QAH state at small $V_{2}$}

Further insight into the tendency toward an interaction-induced QAH phase can be gained from

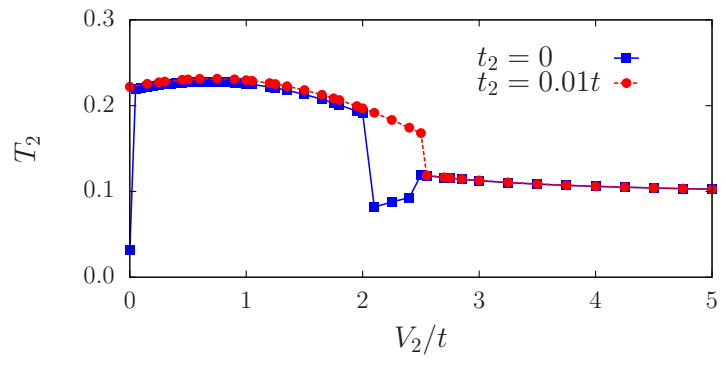

FIG. 7. (Color online) Susceptibility $T_{2}$ defined in Eq. (5) as a function of $V_{2}$. Results were obtained from exact diagonalization of a 24 -site cluster.

Fig. 7. It shows the susceptibility

$$
T_{2}=\frac{1}{L^{2}}\left\langle\left[\sum_{\langle\langle i j\rangle\rangle}\left(e^{\mathrm{i} \phi_{i j}} c_{i}^{\dagger} c_{j}+e^{-\mathrm{i} \phi_{i j}} c_{j}^{\dagger} c_{i}\right)\right]^{2}\right\rangle,
$$

related to the Haldane hopping term [see Eq. [2]], as a function of $V_{2}$, as obtained from exact diagonalization. The phase has been chosen as $\phi_{i j}= \pm \pi / 2$.

We first discuss the case of $t_{2}=0.01 t$, a small symmetry breaking field that establishes a topological QAH state at $V_{2}=0$. Upon switching on the interaction $V_{2}$, the susceptibility $T_{2}$ initially increases. At larger values of $V_{2}$, the susceptibility decreases with increasing $V_{2}$, shows a pronounced drop at around $V_{2}=2.5 t$ and saturates for $V_{2} \geq 2.5 t$. Essentially the same overall behavior is observed in the absence of a symmetry-breaking field, i.e., for $t_{2}=0$. In contrast to $t_{2}=0.01 t$, the ground state for $t_{2}=0$ and $V_{2}=0$ is a semimetal, and $T_{2}$ is much smaller. Around $V_{2}=2.5 t$, we see non-generic signatures related to the cluster considered, as previously discussed for Fig. 2, which are absent on other clusters and for $t_{2}=0.01 t$.

The initial increase of $T_{2}$ with increasing $V_{2}$ may be interpreted as a signature of a tendency toward an interaction-driven QAH phase, in agreement with the fact that a low-energy theory of the model (1) yields an instability of the SM toward the opening of a topological gap via a staggered $t_{2}$ hopping term.24 Moreover, this increase at weak $V_{2}$ is likely to cause the corresponding coupling to increase under a renormalization group flow. Hence, a weak-coupling stability analysis, similar to the one carried out for the interaction-generated quantum spin Hall phase in Ref. 24, would likely indicate ordering tendencies toward this phase. However, the results in Fig. 7 reveal a decrease at larger values of $V_{2}$, in accordance with the absence of a QAH phase at $t_{2}=0$. The absence of such as phase in the model (1), despite the weak-coupling instability, can be attributed to the vanishing of the density of states at the Fermi level in the SM phase, which renders the tendency toward symmetry breaking and spontaneous bond order too weak for a stable phase to exist. However, the enhancement of Haldane-type bond-order correlations for small values of $V_{2}$ suggests that the balance can be tipped in favor of a 
QAH phase, so that the latter may be stabilized in modified or extended models. Weak-coupling instabilities can occur if the density of states at the Fermi level is finite, for example on other two-dimensional lattices 35 or in bilayer systems. $\frac{49159 / 60}{}$

\section{CONCLUSIONS}

We have revisited the problem of spinless fermions on the honeycomb lattice with repulsive, nonlocal interactions. Using exact diagonalization, we found no evidence for the interaction-generated quantum Hall state observed in previous mean-field treatments of the same model ${ }^{24 / 33 / 34}$ Instead, for $V_{1}=0$, our data suggest a direct transition from a correlated semimetal to a gapped, charge-modulated phase at $V_{2} \approx 2.5 t$.

The conclusion regarding the quantum Hall phase is based on the absence of an adiabatic connection to the ground state of the Haldane model throughout the gapped parameter region. The instability of the meanfield quantum Hall state can also be illustrated by including fluctuations around the mean-field solution in the framework of cluster perturbation theory. At smaller $V_{2}$, we found indications for a tendency toward a $\mathrm{QAH}$ state that is enhanced by increasing $V_{2}$, but the vanishing density of states at the Fermi level limits the potential energy gain and thereby prevents the formation of a stable QAH phase. However, the phase may well exist in models with weak-coupling instabilities related to quadratic band crossing points, $\frac{35}{37}$ where the density of states is finite. A weak-coupling quantum Hall phase has also been found at the mean-field level in a model of strained graphene ${ }^{61}$ To identify the modifications of the model which are necessary for the QAH phase to exist represents a fascinating topic for future work.

The charge-modulated phase at large $V_{2}$ turns out to be rather unconventional and was found to have close relations to frustrated spin systems and pinball liquids. It is gapped, but the energy of the lowest-lying excitations becomes independent of the interaction in the strongcoupling regime and is instead determined by the hopping integral $t$. Hence, while the phase clearly emerges from a large $V_{2}$, and therefore is in some sense a Mott insulator, its band gap is set by the hopping integral $t$, a property typical of band insulators.

\section{ACKNOWLEDGMENTS}

We are grateful to F. Assaad, C. Honerkamp, A. Grushin, J. Moore, T. Neupert, S. Rachel, A. Rüegg, J. Venderbos, and M. Vojta for helpful discussions, and acknowledge support from the DFG Grant No. Ho 4489/21 (FOR 1807) and the Emmy Noether Programme. We also thank the authors of Ref. 55 for sharing their results with us prior to publication.

\section{NOTE ADDED}

During the preparation of this manuscript, we learned about the results of Ref. [55, in which the model (1) was studied using exact diagonalization. Both works agree on the absence of an interaction-generated QAH phase.
1 M. Z. Hasan and C. L. Kane, Rev. Mod. Phys. 82, 3045 (2010)

2 X.-L. Qi and S.-C. Zhang, Rev. Mod. Phys. 83, 1057 (2011)

${ }^{3}$ C. L. Kane and E. J. Mele, Phys. Rev. Lett. 95, 146802 (2005)

4 C. L. Kane and E. J. Mele, Phys. Rev. Lett. 95, 226801 (2005)

5 B. A. Bernevig, T. L. Hughes, and S. Zhang, Science 314, 1757 (2006)

6 M. König, S. Wiedmann, C. Brüne, A. Roth, H. Buhmann, L. W. Molenkamp, X.-L. Qi, and S.-C. Zhang, Science 318, 766 (2007)

7 S. A. Parameswaran, R. Roy, and S. L. Sondhi, arXiv:1302.6606

8 E. J. Bergholtz and Z. Liu, Int. J. Mod. Phys. B 27, $1330017(2013)$

9 X. Chen, Z.-C. Gu, and X.-G. Wen, Phys. Rev. B 82, $155138(2010)$

10 X. Chen, Z.-C. Gu, Z.-X. Liu, and X.-G. Wen, Phys. Rev. B 87, 155114 (2013)

${ }^{11}$ X. Chen, Z.-X. Liu, and X.-G. Wen, Phys. Rev. B 84, $235141(2011)$

12 X. Chen, Z.-C. Gu, Z.-X. Liu, and X.-G. Wen, Science 21, 1604 (2012)
13 C. Xu, Phys. Rev. B 87, 144421 (2013)

14 J. Oon, G. Y. Cho, and C. Xu, arXiv:1212.1726

15 F. D. M. Haldane, Phys. Rev. Lett. 61, 2015 (1988)

16 M. Hohenadler and F. F. Assaad, J. Phys.: Condens. Matter 25, 143201 (2013)

Ir S. Rachel and K. Le Hur, Phys. Rev. B 82, 075106 (2010)

18 M. Hohenadler, T. C. Lang, and F. F. Assaad, Phys. Rev. Lett. 106, 100403 (2011)

19 D. Zheng, G.-M. Zhang, and C. Wu, Phys. Rev. B 84, 205121 (2011)

20 C. N. Varney, K. Sun, M. Rigol, and V. Galitski, Phys. Rev. B 82, 115125 (2010)

${ }^{21}$ C. N. Varney, K. Sun, M. Rigol, and V. Galitski, Phys. Rev. B 84, 241105 (2011)

${ }^{22}$ L. Wang, H. Shi, S. Zhang, X. Wang, X. Dai, and X. C. Xie, arXiv:1012.5163

23 A. Rüegg and G. A. Fiete, Phys. Rev. Lett. 108, 046401 (2012)

24 S. Raghu, X. Qi, C. Honerkamp, and S. Zhang, Phys. Rev. Lett. 100, 156401 (2008)

${ }^{25}$ C. Wu and S.-C. Zhang, Phys. Rev. Lett. 93, 036403 (2004)

${ }^{26}$ K. Ohgushi, S. Murakami, and N. Nagaosa, Phys. Rev. B 62, R6065 (2000) 
27 I. Martin and C. D. Batista, Phys. Rev. Lett. 101, 156402 (2008)

28 J. W. Venderbos, M. Daghofer, J. van den Brink, and S. Kumar, Phys. Rev. Lett. 109, 166405 (2012)

29 K.-Y. Yang, W. Zhu, D. Xiao, S. Okamoto, Z. Wang, and Y. Ran, Phys. Rev. B 84, 201104 (2011)

30 A. Rüegg and G. A. Fiete, Phys. Rev. B 84, 201103 (2011)

31 A. Rüegg, C. Mitra, A. A. Demkov, and G. A. Fiete, Phys. Rev. B 85, 245131 (2012)

32 F. Wang and Y. Ran, Phys. Rev. B 84, 241103 (2011)

33 C. Weeks and M. Franz, Phys. Rev. B 81, 085105 (2010)

34 A. G. Grushin, E. V. Castro, A. Cortijo, F. de Juan, M. A. H. Vozmediano, and B. Valenzuela, Phys. Rev. B 87, $085136(2013)$

${ }^{35}$ K. Sun, H. Yao, E. Fradkin, and S. A. Kivelson, Phys. Rev. Lett. 103, 046811 (2009)

36 Q. Liu, H. Yao, and T. Ma, Phys. Rev. B 82, 045102 (2010)

37 J. Wen, A. Rüegg, C.-C. J. Wang, and G. A. Fiete, Phys. Rev. B 82, 075125 (2010)

38 Y. Zhang, Y. Ran, and A. Vishwanath, Phys. Rev. B 79, 245331 (2009)

39 Y. Jia, H. Guo, Z. Chen, S.-Q. Shen, and S. Feng, Phys. Rev. B 88, 075101 (2013)

40 C.-Y. Hou, C. Chamon, and C. Mudry, Phys. Rev. Lett. 98, 186809 (2007)

41 I. F. Herbut, V. Juričić, and B. Roy, Phys. Rev. B 79, $085116(2009)$

42 T. Scaffidi and G. Möller, Phys. Rev. Lett. 109, 246805 (2012)

43 P. Zanardi and N. Paunković, Phys. Rev. E 74, 031123 (2006)

44 W.-L. You, Y.-W. Li, and S.-J. Gu, Phys. Rev. E 76, 022101 (2007)
45 More precisely: Between phases whose Chern numbers differ by an odd number.

${ }^{46}$ Except for the point $t_{2}=V_{1}=V_{2}=0$, where the Dirac semimetal is six-fold degenerate.

47 A. Liebsch, Phys. Rev. Lett. 111, 029701 (2013)

48 M. M. Scherer, S. Uebelacker, D. D. Scherer, and C. Honerkamp, Phys. Rev. B 86, 155415 (2012)

49 M. M. Scherer, S. Uebelacker, and C. Honerkamp, Phys. Rev. B 85, 235408 (2012)

${ }^{50}$ L. Cano-Cortés, A. Ralko, C. Février, J. Merino, and S. Fratini, Phys. Rev. B 84, 155115 (2011)

51 G. Wannier, Phys. Rev. B 79, 357 (1950)

52 C. Hotta and N. Furukawa, Phys. Rev. B 74, 193107 (2006)

53 S. Morohoshi and Y. Fukumoto, J. Phys. Soc. Jpn. 77, 023708 (2008)

54 S. Kourtis and M. Daghofer, arXiv:1305.6948

55 N. A. García-Martínez, A. G. Grushin, T. Neupert, B. Valenzuela, and E. V. Castro, arXiv:1308.6094

56 D. Sénéchal, D. Perez, and D. Plouffe, Phys. Rev. B 66, 075129 (2002)

57 M. Aichhorn, H. G. Evertz, W. von der Linden, and M. Potthoff, Phys. Rev. B 70, 235107 (2004)

58 A real order parameter $\chi_{i j}$ with opposite sign on the two sublattices likewise opens a gap which is, however, topologically trivial.

59 O. Vafek, Phys. Rev. B 82, 205106 (2010)

60 F. Zhang, H. Min, M. Polini, and A. H. MacDonald, Phys. Rev. B 81, 041402 (2010)

${ }^{\circ 1}$ B. Roy and I. F. Herbut, Phys. Rev. B 88, 045425 (2013) 